\title{
State-dependent Preferences in Prediction Markets and Prices as Aggregate Statistic
}

\begin{abstract}
Urmee Khan ${ }^{1}$
Abstract

If traders in prediction markets have state-dependent preferences so that marginal utility of money varies across states, prices in a Rational Expectation equilibrium are quantile statistics of distributions that derive from both the distribution of realized signals, and the distribution of state-dependence parameters. As a result, even with a common prior and regardless of whether prices reveal realized signals fully or not, the interpretation of prices as posterior probabilities remains problematic.
\end{abstract}

Keywords: Prediction Markets, Information Aggregation, State-dependent Preferences

\section{Introduction}

Prediction markets have become popular in the recent years as a tool for aggregating information spread across individuals. The use of such markets as an aid to decision making within large corporations is growing. These markets are expected to aggregate information efficiently and generate predictions of future events. Various theoretical issues with regards to the efficiency of these markets have been investigated. Notable among them are heterogenous prior beliefs of traders (Manski [2006], Ottaviani et al. [2015]), wealth effects (Ottaviani et al. [2015]), possibility of price manipulation and outcome manipulation by traders (Ottaviani and Sørensen [2007]), and feedback effects (Lieli and Nieto-Barthaburu [2009]). This note looks at another important but hitherto neglected issue, that of traders having state-dependent preferences. In the context of prediction markets, which are primarily an elicitation mechanism of traders' subjective beliefs about an uncertain event of importance, it is very plausible that traders have preferences over the realized outcome itself, and this would complicate the process of elicitation of 'pure' beliefs (or the private information they have pertaining purely to the likelihood of an outcome) and detangle it from the traders' state preferences (or private information that is payoff relevant upon a particular realization, but not relevant to the likelihood of the outcome itself). Elicitation of priors in the context of state-dependent preferences is an open problem (see for eg.,Karni [2015] and the references therein). In prediction markets, which are modelled on theoretical Arrow-Debreu asset markets, such state-dependent preferences would

\footnotetext{
${ }^{1}$ University of California, Riverside. I am grateful to Max Stinchcombe and Robert Lieli for many useful discussions and to the Editor for valuable suggestions on the exposition.
} 
induce an insurance motive for trading, and it is not clear how resultant equilibrium prices are to be interpreted as a measure of aggregate market information. We investigate to what extent prices as a statistic relate to the distribution of realized signals (Wolfers and Zitzewitz [2006], Manski [2006]).

\section{Brief Overview of Related Literature}

Information aggregation in markets via the price mechanism has been a central topic of research in Economics for a long time. Hayek [1945] argued that prices are the way decentralized 'knowledge' gets aggregated in markets. Prediction markets are one of the most recent examples of market-based mechanisms designed to aggregate information that is held privately by individual agents, with a view to using them for predicting real events. With regards to the general question of the 'efficiency' of markets in aggregating information, there are two strands of theoretical literature. The two are distinct in methodology, but conceptually related. One of them pertains to the 'Rational Expectations' paradigm (Radner [1979] is an early classic). One of the most influential papers in this strand is Milgrom and Stokey [1982], which establishes the following result: with common priors and concordant beliefs, no trade will occur in a Rational Expectations equilibrium purely based on differences in private information, if the equilibrium price is fully revealing. This striking finding about the implications of a fully revealing price in a Rational Expectations setting has inspired a very large body of literature, with researchers attempting to build models that could explain the real world trading patterns in financial markets. Heterogenous priors have been an important and popular assumption in circumventing the starkness of the no-trade theorem. Morris [1994] shows that the extent of information aggregation that takes places in a market with heterogeneous priors depend on the trading environment. The particulars of the trading enviornment have been the concern of the second strand of literature; there is another large body of research that investigate the interplay of market efficiency and market microstructure. Kyle [1985] is an early classic, and this strand of literature is very active and vibrant to this day, encompassing both theoretical and empirical approaches(see, for eg., O'hara [1995] for the thoery, and Biais et al. [2005] for a survey of empirical and policy literature).

Prediction markets as a particular implementation of the market efficiency idea has also generated a significant amount of theoretical and empirical interest (see Arrow et al. [2008]for a statement by prominent economists about the promise of prediction markets). The introduction mentions the papers most pertinent to the focus of this note. Much of the theoretical work fall within one of the two strands mentioned above. An important set of questions pertain to the difference between prediction markets and other financial markets in one significant aspect, namely, that traders may have preferences and objectives that are not captured only by the financial payout of the asset itself. Examples 
include signaling motives, or direct preferences over the events themselves. When, and to what extent, the prices realized in the prediction markets can be interpreted as 'estimated aggregate probabilities' of the events of interest is of prime importance. At this moment, this set of questions are quite open and under active research.

\section{Model}

The model and the solution techniques follow those in Manski [2006] and closely mirror Ottaviani et al. [2015].

Let there be an unit measure of traders in a prediction market designed to predict a binary event $A, A^{c}$. The market trades in a binary asset, asset $A$ pays off 1 if event $A$ happens, and 0 otherwise, while asset $A^{c}$ pays off 1 if $A^{c}$ happens, and 0 otherwise ${ }^{2}$. Traders obtain each unit portfolio (consisting of one unit of assets $A$ and $A^{c}$ each) for $\$ 1$ from the market maker and trade among themselves in individual assets. We thus normalize the supply of each asset to 1 . Market also stands ready to buy back each unit portfolio for $\$ 1$ at any given time. Thus the unit portfolio is a riskless asset, and arbitrage opportunities are eliminated, since the combined trading price of the two assets together would always be 1 . We assume each trader $i$ has wealth $w_{i}$. Traders are not allowed to hold negative amounts of assets (i.e. 'selling short'is ruled out)

Traders have state-dependent preferences over money, so that marginal utility is different in different state realizations. Formally, trader $i$ has utility function $u_{i}(m, j)$ where $m$ is the amount of money holding and $j$ is the state i.e outcome realization, $A$ or $A^{c}$. Contingent on state realization, traders are risk neutral. To keep the model as simple as possible, we assume the following functional form:

$$
u_{i}(m, A)=\theta_{i} m_{i}
$$

and

$$
u_{i}\left(m, A^{c}\right)=m_{i}
$$

$\theta_{i}$ are i.i.d random variables taking values in $(0, \infty)$ with a continuous and strictly increasing distribution function $G(\theta)$; it captures the ratio of marginal utilities of money between the two states, arising out of the individual's preferences over the state realization separate from the monetary gain. The signal $s_{i}$ and state-dependence parameter $\theta_{i}$ are assumed to be independent.

Traders have common prior over the two states: $P(A)=\pi$ and $P\left(A^{c}\right)=1-\pi$. Each trader receives an i.i.d private signal $s_{i}$, with continuous distribution $F(s)$ on a signal space $S$. They form posterior beliefs over states, and the aim of a prediction market is aggregate these posteriors, the equilibrium price being

\footnotetext{
${ }^{2}$ The institutional details follow closely the Iowa Electronic Markets
} 
the aggregator statistic. The following is an investigation of what this statistic might mean in the presence of state dependent utilities.

\section{Individual Trader's Maximization Problem}

Let's consider each individual risk-neutral trader's maximization problem. Given risk neutrality and the linearity of the utility function over money, the optima are corner solutions. A trader would invest all her money in an asset if the expected payout of the asset, with her updated beliefs given the price and her own signal realization, exceeds the price of the asset, and would invest none in the converse case.

No-arbitrage condition would imply that prices of both assets can be normalized to 1 . We denote price of $A$ as $p$ and that of $A^{c}$ as $1-p$.

Let the posterior beliefs of trader $i$ be denoted by $q\left(A \mid s_{i}\right)$ and $1-q\left(A \mid s_{i}\right)$ and the 'asset position' chosen by the trader be $\left(y_{A_{i}}, y_{A_{i}^{c}}\right)$. With Arrow-Debreu securities, $\left(y_{A_{i}}, y_{A_{i}^{c}}\right)$ are also the event dependent cash payouts.

Before trading, each trader observes her private signal $s_{i}$. Her posterior is formulated by

$$
\frac{q_{i}}{1-q_{i}}=\frac{\pi}{1-\pi} L_{i}
$$

Here $L_{i}$ is the likelihood function $\frac{f\left(s_{i} \mid A\right)}{f\left(s_{i} \mid A^{c}\right)}$ for trader $i$.

So the agent is maximizing, after observing her own signal $s_{i}$

$$
\left[q\left(A \mid s_{i}\right)-p\right] \theta_{i} y_{A_{i}}+\left[1-q\left(A \mid s_{i}\right)-(1-p)\right] y_{A_{i}^{c}}
$$

Given market price $p$, the subjective expected return on asset $A$ is $\theta_{i}\left[q\left(A \mid s_{i}\right)-p\right]$, and the expected return on the other asset is $1-q\left(A \mid s_{i}\right)-(1-p)=p-q\left(A \mid s_{i}\right)$. Given the restriction on negative holdings, if $q\left(A \mid s_{i}\right)>p$, trader $i$ invests all her money in asset $A$, i.e, exchanges out her entire holding of asset $A^{c}$ for $\frac{(1-p) w_{i}}{p}$ units of $A$. Her asset position is then $\left(y_{A_{i}}, y_{A_{i}^{c}}\right)=\left(\frac{w_{i}}{p}, 0\right)$. Conversely, if $q\left(A \mid s_{i}\right)<$ $p$, her portfolio is $\left(y_{A_{i}}, y_{A_{i}^{c}}\right)=\left(0, \frac{w_{i}}{1-p}\right)$

\section{Equilibria and Results}

We look at Rational Expectations Equilibria in this market. Several observations are in order. Given state-dependent preferences, the ex-ante allocation is not Pareto-optimal necessarily, so no-trade theorem is not an issue. Also, observe that that traders are facing additional uncertainty in the market in the 
form of the unknown state-bias parameters of other market participants, on top of the uncertainty regarding the state. The binary assets now do not provide complete contingent markets any more. Hence the existence of a perfectly revealing price equilibrium is not guaranteed.

Let $\theta$ and $s$ be a profile of bias parameters and a profile of signals respectively. A Rational Expectation Equilibrium would constitute of the following components, such that traders maximize their expected utility and markets clear:

- A price function $p(s, \theta)$

- demand functions for each individual trader, $y_{i}\left(s_{i}, \theta_{i}, p\right)$

We consider both the non-revealing and fully revealing price equilibria. Note that a 'fully-revealing' price equilibrium in this case is still in the traditional sense, i.e a price function that aggregates private information about the likelihood of the states, nothing more. Here we do not get into the questions of under what conditions a revealing or non-revealing equilibrium will arise, instead we focus on what the price as a statistic would tell us in each case.

Formally, A non-revealing price function $p(s, \theta)$ is such that,

$$
E\left(u_{i} \mid s_{i}\right)=E\left(u_{i} \mid p\right)
$$

In other words, traders' observation of market price do not lead to any refinement of the posterior that was formulated with the private signals alone (Allen and Jordan [1998]). Note that given the independence of $\theta$ and $s_{i}$, conditioning on $\theta$ is superfluous.

The optimality condition for individual traders in such an equilibrium is the following: risk neutral traders invest all their money in asset $A$ if

$$
\frac{\theta_{i} q\left(A \mid s_{i}\right)}{1-q\left(A \mid s_{i}\right)} \geq \frac{p}{1-p}
$$

Recall $L_{i}$, the likelihood function for the private signal of trader $i$,

$$
L_{i}=\frac{f\left(s_{i} \mid A\right)}{f\left(s_{i} \mid A^{c}\right)}
$$

Condition (1) then can be written as

$$
\theta_{i} L_{i} \frac{\pi(A)}{1-\pi(A))} \geq \frac{p}{1-p}
$$

This can be rewritten as,

$$
\theta_{i} L_{i} \geq \frac{p}{(1-p)} \frac{(1-\pi(A))}{\pi(A))}
$$


Let $x_{i}=\theta_{i} L_{i}$ and denote its i.i.d distribution as $H(x)$. Given our independence assumptions $H(x)$ can be derived in a straightforward fashion from $F(s)$ and $G(\theta)$. Given aggregate wealth is normalized to $1, H(x)$ would be the proportion of total wealth held by traders for whom $\theta_{i} L_{i} \leq x$. This is exactly the proportion of wealth held by people, who demand $A^{c}$ in positive amounts, $w_{i} / 1-p$. So, the aggregate demand for, $A^{c}$,

$$
\bar{A}^{c}=\frac{H\left(\frac{p}{(1-p)} \frac{(1-\pi(A))}{\pi(A)}\right)}{1-p}
$$

Which can be explicitly seen from, given the above discussion,

$$
\bar{A}^{c}=\frac{1}{1-p}\left[\int_{0}^{\frac{p}{(1-p)} \frac{(1-\pi(A))}{\pi(A)}} d H(x)\right]
$$

Market equilibrium price can then be characterized by the market clearing condition, as the aggregate supply for each asset if normalized to 1:

$$
\frac{H\left(\frac{p}{(1-p)} \frac{(1-\pi(A))}{\pi(A)}\right)}{1-p}=1
$$

Which gives us,

$$
\frac{1-H\left[\frac{p}{(1-p)} \frac{(1-\pi)}{\pi}\right]}{p}=1
$$

Hence the equilibrium price is a quantile statistic of the joint distribution of the signal likelihood functions and the bias parameters.

We now show that the essential entanglement between the bias parameters and the signals captured in the price statistic holds true, even if the market price reveals the private signals to the full extent. In such a 'fully revealing' equilibrium,

$$
E\left(u_{i} \mid p\right)=E\left(u_{i} \mid s\right)
$$

where $s$ is the full realized signal profile. The implication is that, in such a price equilibrium, the posterior beliefs are equated across traders (note that trade would now be purely insurance motivated).

The analogue to equation (2) would now be

$$
\theta_{i} L \geq \frac{p}{(1-p)} \frac{(1-\pi(A))}{\pi(A))}
$$

Where $L$ is now the common likelihood function for all traders, i.e $L=f(s \mid A)$ 
Now we get a price determination equation

$$
\frac{1-G\left[\frac{p}{(1-p)} \frac{(1-\pi)}{\pi} \frac{1}{L}\right]}{p}=1
$$

This condition has the same flavor to the one in Ottaviani et al. [2015] where the heterogeneity is in the prior beliefs, whereas here the heterogeneity is due to the idiosyncratic state-dependent preferences. It shows that although equilibrium price would be an increasing function of the aggregate realized likelihood across all traders, the price is a quantile statistic of the distribution of the bias parameter. Further research would aim to derive a full set of results that examine in detail the various aspects of price dynamics in this setting, and the implications of other factors of interest including risk aversion and wealth effects.

\section{Conclusion}

In this paper, we present a simple model of a prediction market where traders have state-dependent preferences. Given that state-dependence manifests in differing marginal utilities across states, Rational Expectation Equilibrium prices, whether revealing or non-revealing, turn out to be quantile statistics of a distribution based on not only the realized set of signals, but also the state-dependence parameters of the traders. In other words, speculative trade and insurance trade get entangled in the price formation process, and make probabilistic interpretations of prices problematic.

\section{References}

Beth Allen and James S Jordan. The existence of rational expectations equilibrium: a retrospective. Organizations with Incomplete Information, pages 42-60, 1998.

K. J. Arrow, R. Forsythe, M. Gorham, R. Hahn, R. Hanson, J. O. Ledyard, S. Levmore, R. Litan, P. Milgrom, and F. D. Nelson. Economics: The promise of prediction markets. Science, 320(5878):877, 2008. ISSN 0036-8075.

B. Biais, L. Glosten, and C. Spatt. Market microstructure: A survey of microfoundations, empirical results, and policy implications. Journal of Financial Markets, 8(2):217-264, 2005.

F. A. Hayek. The use of knowledge in society. The American Economic Review, pages 519-530, 1945.

Edi Karni. A mechanism for eliciting sets of priors, 2015. 
A. S. Kyle. Continuous auctions and insider trading. Econometrica: Journal of the Econometric Society, 53(6):1315-1335, 1985.

Robert P Lieli and Augusto Nieto-Barthaburu. Feedback in prediction markets. Technical report, 2009.

Charles $\mathrm{F}$ Manski. Interpreting the predictions of prediction markets. economics letters, 91(3):425-429, 2006.

Paul Milgrom and Nancy Stokey. Information, trade and common knowledge. Journal of Economic Theory, 26(1):17-27, 1982.

Stephen Morris. Trade with heterogeneous prior beliefs and asymmetric information. Econometrica: Journal of the Econometric Society, pages 1327-1347, 1994.

Maureen O'hara. Market microstructure theory, volume 108. Blackwell Cambridge, MA, 1995.

Marco Ottaviani and Peter Norman Sørensen. Outcome manipulation in corporate prediction markets. Journal of the European Economic Association, 5 (2-3):554-563, 2007.

Marco Ottaviani, Peter Norman Sørensen, et al. Price reaction to information with heterogeneous beliefs and wealth effects: Underreaction, momentum, and reversal. American Economic Review, 105(1):1-34, 2015.

R. Radner. Rational expectations equilibrium: Generic existence and the information revealed by prices. Econometrica: Journal of the Econometric Society, 47(3):655-678, 1979.

Justin Wolfers and Eric Zitzewitz. Interpreting prediction market prices as probabilities. Technical report, National Bureau of Economic Research, 2006. 